\title{
soShearEnergy of permutation of necklace
}

\author{
J. Chithra ${ }^{* *}$ and S.P. Jeyakokila ${ }^{2}$
}

\begin{abstract}
Pnueli, Lempel and Even introduced Permutation Graphs in 1971 [8]. If $i, j$ belong to a permutation on $p$ symbols $\{1,2, \ldots, p\}$ and if $i$ is less than $j$ then there is an edge between $i$ and $j$ in the permutation graph if $i$ appears after $j$ in the corresponding sequence. A subset $D$ of $A$, whose closed neighborhood is $A$ in $\pi$ is a dominating set of $\pi$. Energy of a graph with respect to the minimal dominating set was introduced by B.D.Acharyaetal [4]. soShearEnergy is introduced in [5]. In this paper necklace graph is realized as a permutation graph and soShearEnergy of permutation of necklace is calculated with respect to minimal dominating set.
\end{abstract}

\section{Keywords}

Permutation graphs, Necklace, Domination of first kind and second kind, soShearEnergy.

AMS Subject Classification

05C30, 05C50, 05C62, 05C69, 05C85.

1,2 Department of Mathematics, Lady Doak College, Madurai-625002, India.

*Corresponding author: ${ }^{1}$ chithra@ldc.edu.in ${ }^{2}$ jeyakokila@ldc.edu.in

Article History: Received 21 December 2018; Accepted 11 February 2019

(C)2017 MJM.

\section{Contents}

1 Introduction ................................. 32

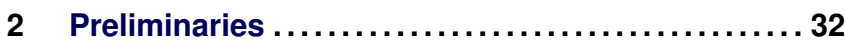

3 Permutation realising a Necklace $\ldots \ldots \ldots \ldots \ldots \ldots, 33$

4 soShearEnergy of permutation of Necklace ....... 34

5 Energy curves of Necklace ..................... 35

6 soShearEnergy of permutation of a necklace......35

References .

35

\section{Introduction}

Number of researches are carried out by the computer scientists all over the world since the concept is introduced and hence many notions of graphs in permutation graphs are modeled using polynomial time algorithms. Another way of defining the permutation graph is available in the literature. Harary and Chartrand defined a permutation graph as: for any permutation $\alpha$ in $S_{p}$, the $\alpha$-permutation graph of a labeled graph $G$ is the union of two disjoint copies $G_{1}$ and $G_{2}$ of $G$ together with the lines joining point $v_{i}$ of $G_{1}$ with $v_{\alpha(i)}$ of $G_{2}$ [1]. Both the kinds are unrelated. The study on the permutation graph introduced by Pnueli,Lempel and Even stimulated us to establish measures to find the domination numbers of the permutations corresponding to the permutation graphs with the perspective of graph theory. Our paper deals with the permutation graph defined by Pnueli,Lempel and Even. An algorithm is developed by J.Chithra and S.P.Subbiah [2] to determine the minimal dominating sets for permutations and thus for permutation graphs. Energy of a graph was developed during 1970. Different types of energies were evolved from different matrices. Latter B.D.Acharya et al. introduced Energy of a graph of a dominating set . soShearEnergy of a graph with respect to the given minimal dominating set is introduced by S.P.Jeyakokila and P.Sumathi and an algorithm is also developed by them. In this article the graph named as necklace is realized by a permutation for which the soShearEnergy is determined. The results are found to be interesting since they integrate permutations, graphs and energy of graphs.

\section{Preliminaries}

Definition 2.1. Let $\pi$ be a permutation on a finite set $V=$ $\left\{a_{1}, a_{2}, a_{3}, \ldots, a_{p}\right\}$ given by $\pi=\left(\begin{array}{ccccc}a_{1} & a_{2} & a_{3} & \ldots & a_{p} \\ a_{1}^{\prime} & a_{2}^{\prime} & a_{3}^{\prime} & \ldots & a_{p}^{\prime}\end{array}\right)$ where $\left|a_{i+1}-a_{i}\right|=c>0,1 \leq i \leq p-1$. The sequence of $\pi$ is given by $s(\pi)=\left\{a_{1}^{\prime}, a_{2}^{\prime}, a_{3}^{\prime}, \ldots, a_{p}^{\prime}\right\}$.

Definition 2.2. Let $a_{i}, a_{j} \in V$. Then the residue of $a_{i}$ and $a_{j}$ in $\pi$ is denoted by $\boldsymbol{R e s}\left(a_{i}, a_{j}\right)$ and is given by $\left(a_{i}-a_{j}\right)\left(\pi^{-1}\left(a_{i}\right)-\right.$ $\left.\pi^{-1}\left(a_{j}\right)\right)$.

Definition 2.3. When elements of $V$ are ordered in $L_{1}$ and the sequence of $\pi$ are represented in $L_{2}$, then a line joining 
$a_{i}$ in $L_{1}$ and $a_{i}$ in $L_{2}$ is represented as $l_{i}$. This is known as line representation of $a_{i}$ in $\pi$ [9]. Let $l_{i}$ and $l_{j}$ are the lines corresponding to the elements $a_{i}$ and $a_{j}$ respectively in $\pi$. Then $l_{i}$ crosses $l_{j}$ if $\operatorname{Res}\left(a_{i}, a_{j}\right)<0$.

Definition 2.4. Let $\pi$ be a permutation on a finite set $V=$ $\left\{a_{1}, a_{2}, a_{3}, \ldots, a_{p}\right\}$ given by $\pi\left(a_{i}\right)=a_{i}^{\prime}, 1 \leq i \leq p$ where $\mid a_{i+1}-$ $a_{i} \mid=c, c>0,1 \leq i \leq p-1$. Then the Permutation Graph $G_{\pi}$ is given by $G_{\pi}=\left(V_{\pi}, E_{\pi}\right)$ where $V_{\pi}=\left\{a_{1}, a_{2}, \ldots, a_{p}\right\}$ and $a_{i} a_{j} \in E_{\pi}$, if $\operatorname{Res}\left(a_{i}, a_{j}\right)<0$. If $l_{i}$ crosses $l_{j}$ then $\left(a_{i}, a_{j}\right) \in E_{\pi}$.

Example 2.5. Let $\pi$ be a permutation on a finite set $V=$ $\{1,2,3,4,5\}$ given by $\pi(1)=3, \pi(2)=5, \pi(3)=1, \pi(4)=4$ and $\pi(5)=2$. (i.e) $\pi=\left(\begin{array}{lllll}1 & 2 & 3 & 4 & 5 \\ 3 & 5 & 1 & 4 & 2\end{array}\right)$.

Then the line $l_{1}$ crosses $l_{3}$ and $l_{5} ; l_{2}$ crosses $l_{3}, l_{4}$, and $l_{5}$; $l_{3}$ crosses $l_{1}$ and $l_{2} ; l_{4}$ crosses $l_{2}$ and $l_{5} ; l_{5}$ crosses $l_{1}, l_{2}$ and $l_{4}$.

Definition 2.6. A graph $G$ is a permutation graph if there exists $\pi$ such that $G_{\pi} \cong G$. (i.e) a graph is a permutation graph if it is realizable by a permutation $\pi$. Otherwise it is not a permutation graph.

Example 2.7. Let $\pi=\left(\begin{array}{lllll}1 & 2 & 3 & 4 & 5 \\ 3 & 5 & 1 & 4 & 2\end{array}\right)$.

Then $G_{\pi}=\left(V_{\pi}, E_{\pi}\right)$ where $V_{\pi}=\{1,2,3,4,5\}$ and $E_{\pi}=\{(1,3),(1,5),(2,3),(2,4),(2,5),(4,5)\}$.

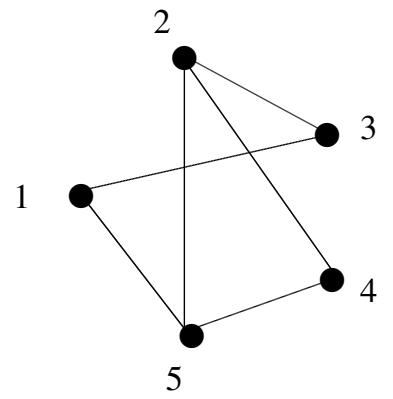

Definition 2.8. Let $G$ be a graph and $S$ be a subset of $V(G)$. Let $v \in V-S$. The idegree of $v$ with respect to $S$ is the number of neighbours of $v$ in $V-S$ and it is denoted by $i d_{S}(v)$.

Definition 2.9. Let $G$ be a graph and $S$ be a subset of $V(G)$. Let $v \in V-S$. The odegree of $v$ with respect to $S$ is the number of neighbours of $v$ in $V-S$, in the set $S$ and is denoted as $\operatorname{od}_{S}(v)$.

Definition 2.10. Let $G$ be graph and $S$ be a subset of $V(G)$. Let $v \in V-S$. The oidegree of $v$ with respect to $S$ is od $\operatorname{od}_{S}(v)-$ $i d_{S}(v)$ if $o_{s}>i d_{s}$ and it is denoted by oid $(v)$

Definition 2.11. Let $G$ be a graph and $D$ be a minimal dominating set. oShearEnergy of a graph with respect to $D$ denoted by $\operatorname{os}_{D}(G)$ is the sum of all oid if $o d_{s}>i d_{s}$, zero otherwise.
Definition 2.12. Let $G$ be a graph and $D$ be a minimal dominating set, then ShearEnergy curve is the curve obtained by joining the oShearEnergies of $G$ with respect to $D_{i}$ for $0 \leq i \leq n-1$, marked off by taking the number of vertices of $D_{i}$ along the $X$-axis and the oShearEnergies along the $Y$-axis

Definition 2.13. Let $G$ be a graph and $D$ be a minimal dominating set. soShearEnergy of a graph with respect to $D$ is $|V-D|$

$\sum_{i=0}$ os $\varepsilon_{D_{i}}(G)$ where $D_{i+1}=D_{i} \cup V_{i+1}$, and $V_{i+1}$ is a singleton set with minimum oidegree of $V-D_{i}$ and $D_{0}$ is a minimal dominating set where $0 \leq i \leq|V-D|$. It is denoted by $\operatorname{sos}_{D}(G)$.

\section{Permutation realising a Necklace}

Definition 3.1. An n-point contact of $K_{2}$ is $N K_{n}$ (i. e) $N K_{n}=$ $K_{2}+n K_{1}$ along with an edge $K_{2}=u v$ is called a necklace with rope $u v$ and is denoted by $N K_{n}$ with rope $u v$. Here $n$ denotes number of beads, where a bead is an element of degree 2 in $N K_{n}$. [Ref:[3]]

Theorem 3.2. Let $\pi$ be a permutation on $p$ symbols $V=$ $\left\{a_{1}, a_{2}, \ldots, a_{p}\right\}$ given by $\pi\left(a_{i}\right)=a_{i}^{\prime}, 1 \leq i \leq p$, where $\mid a_{i+1}-$ $a_{i} \mid=c, c>0$. Then a transposition $\left(a_{i} a_{j}\right), i<j$, realizes $a$ permutation graph which is a subgraph of $G_{\pi}$ isomorphic to a Necklace with the edge $a_{i} a_{j}$ as a rope and with $n=a_{i}-a_{j}-1$ number of beads, as a component and other elements are isolates.

Proof.

Let $\pi=$

$\left(\begin{array}{cccccccccc}a_{1} & a_{2} & \ldots & a_{i} & a_{i+1} & \ldots & a_{j} & a_{j+1} & \ldots & a_{p} \\ a_{1} & a_{2} & \ldots & a_{j} & a_{i+1} & \ldots & a_{i} & a_{j+1} & \ldots & a_{p}\end{array}\right)$.

The transposition $\left(a_{i} a_{j}\right)$ is given by

$\left(a_{i} a_{j}\right)=\left(\begin{array}{ccccc}a_{i} & a_{i+1} & a_{i+2} & \ldots & a_{j} \\ a_{j} & a_{i+1} & a_{i+2} & \ldots & a_{i}\end{array}\right)$.

Here $l_{i}$ crosses $l_{i+1}, l_{i+2}, l_{i+3}, \ldots, l_{j}$ and

$l_{j}$ crosses $l_{j-1}, l_{j-2}, l_{j-3}, \ldots, l_{i}$.

Therefore $a_{i}$ and $a_{j}$ are of degree $a_{j}-a_{i}$.

All $l_{i+1}, l_{i+2}, \ldots, l_{j-1}$ cross $l_{i}$ and $l_{j}$.

Hence $a_{i+1}, a_{i+2}, \ldots, a_{j-1}$ are of degree 2 .

Hence they are bead vertices and they are $a_{j}-a_{i}-1$ in number.

Therefore by Definition $3.1\left(a_{i} a_{j}\right)$ realizes a necklace $N K_{n}$ with $a_{i} a_{j}$ as a rope and with $n=a_{i}-a_{j}-1$ number of beads.

As other elements are their own images they are isolates.

Hence the proof.

Example 3.3. Let $\pi$ be a permutation on $p$ symbols $V=$ $\left\{a_{1}, a_{2}, \ldots, a_{p}\right\}$ given by $\pi\left(a_{i}\right)=a_{i}^{\prime}, 1 \leq i \leq p$, where $\mid a_{i+1}-$ $a_{i} \mid=c, c>0$ and

$\pi=\left(\begin{array}{llllll}a_{i} & a_{i+1} & a_{i+2} & a_{i+3} & a_{i+4} & a_{i+5} \\ a_{i+5} & a_{i+1} & a_{i+2} & a_{i+3} & a_{i+4} & a_{i}\end{array}\right)=\left(a_{i} a_{i+5}\right)$. 


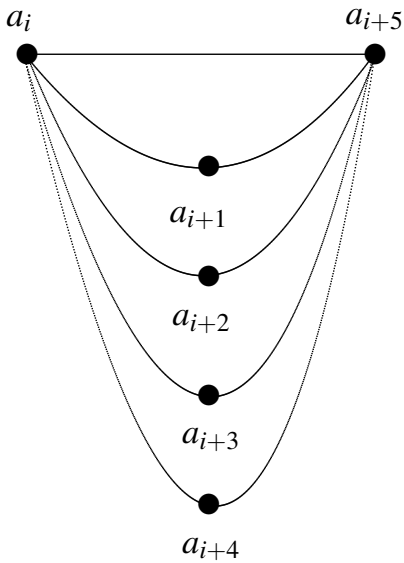

$a_{1}$

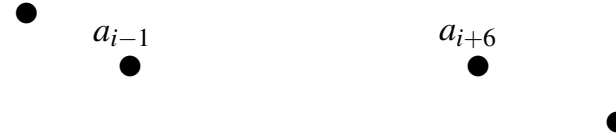

$N K_{4}$ with rope $a_{i} a_{i+5}$

\section{4. soShearEnergy of permutation of Necklace}

Definition 4.1. Two minimal Dominating sets are possible for $\mathrm{NK}_{m}$. Let us name the two minimal dominating sets as domination of first kind and domination of second kind as follows.

(i) Domination of first kind denoted by ${ }^{1} D$ is the minimal dominating set with one of the vertices in the rope.

(ii) Domination of second kind denoted by ${ }^{2} D$ is the minimal dominating set containing vertices of the beads.

Lemma 4.2. Let $N K_{m}$ be the Necklace, then

(i) $(a) \operatorname{sos} \varepsilon_{N K_{m}}\left({ }^{1} D\right)=n^{2}+n+1$ where $n=\frac{m}{2}$ when $m$ is even

(b) $\operatorname{sos} \varepsilon_{N K_{m}}\left({ }^{1} D\right)=(m+1)+\varepsilon_{N K_{m-2}}\left({ }^{1} D\right)$ when $m$ is odd, and $\operatorname{sos} \varepsilon_{N K_{1}}\left({ }^{1} D\right)=1$.

(ii) $\operatorname{sos} \varepsilon_{N K_{m}}\left({ }^{2} D^{c}\right)=3 m-1$.

Proof. Let $N K_{m}$ be the given necklace graph.

(i) Let the dominating set ${ }^{1} D$ be of first kind. Then the dominating set ${ }^{1} D$ is the singleton set with vertex which is adjacent to all other vertices of the necklace. Then the set $V-{ }^{1} D$ contains one vertex of the rope and m number of beads.

Case(i) Let $m$ be even.

At the initial stage, odegree of all the vertices is 1 and idegree of all the bead vertices is 1 and the vertex of rope is $m-2$. Hence $\operatorname{os} \varepsilon_{D_{1}}\left(N K_{m}\right)$ is zero for the $\frac{m}{2}-1$ number of stages. At the $\frac{m}{2}$ nd stage, odegree of the vertex of the rope is $\frac{m}{2}$ since $\frac{m}{2}$ vertices are shifted to the dominating set.

Hence oid of that vertex is 1 .

At the $\frac{m}{2}+1$ th stage, the vertex of the rope is shifted to the dominating set.

The remaining vertices of $V-{ }^{1} D$ are independent and hence the idegree of all the vertices are zero and odegree of the vertices are 2 .

Hence at this stage $o s \varepsilon_{\frac{m}{2}+1}\left(N K_{m}\right)=2 \frac{m}{2}=m$.

Since $\mathrm{m}$ is even, let us consider $\mathrm{m}=2 \mathrm{n}$, then $\operatorname{os} \varepsilon_{D_{\frac{m}{2}+1}}\left(N K_{m}\right)=$ $2 n$.

The remaining vertices are shifted one by one till cardinality of $V-{ }^{1} D$ is zero, so that the $o s \varepsilon_{D}\left(N K_{m}\right)\left({ }^{1} D\right)$ decreases 2 by 2 till the cardinality reaches zero. Therefore $\operatorname{sos}_{1} D\left(N K_{m}\right)=$ $1+2 n+2(n-1)+\cdots+2+0$ On simplification, $\operatorname{sos}_{N K_{m}}\left({ }^{1} D\right)=n^{2}+n+1$ where $n=\frac{m}{2}$.

Case(ii)

Let $\mathrm{m}$ be odd.

When $m=1$, the dominating set contains a vertex of degree 2 which is one of the vertices of the rope. Vertices in the set $V-{ }^{2} D$ are vertices of rope of degree 2 and a bead vertex is also of degree 2 .

Hence odegree and idegree of both the vertices are 1 .

Therefore at the first stage oidegree of both the vertices are zero and $o s \varepsilon_{1} D_{1}\left(N K_{3}\right)\left({ }^{1} D\right)=0$.

At the second stage the vertex of the rope is shifted to the dominating set and hence idegree of the remaining vertex is zero and odegree is 2 .

Hence $\operatorname{os}_{1} \varepsilon_{D_{2}}\left(N K_{3}\right)=2$.

${ }^{o s} \varepsilon_{1} D\left(N K_{m}\right)$ attains integer value only at $\left\lceil\frac{m}{2}\right\rceil$ nd stage. 
Hence $\operatorname{os} \varepsilon_{D_{\left\lceil\left\lceil\frac{m}{2}\right\rceil\right.}}\left(N K_{m}\right)$ is $(\mathrm{m}+1)$. And it is the repetition of necklace of size $m-1$ after this stage.

Hence $\operatorname{sos} \varepsilon_{N K_{m}}\left({ }^{1} D\right)=(m+1)+\varepsilon_{N K_{m-2}}\left({ }^{1} D\right)$ when $\mathrm{m}$ is odd, $\operatorname{sos}_{N K_{1}}\left({ }^{1} D\right)=1$.

(ii) Let the dominating set be of second kind, that is, the dominating set contains the bead vertices. Hence the $V-{ }^{2} D$ set contains the vertices of ropes for all values of $m$.

As they are adjacent to each other the idegree of both the vertex is 1 .

They are also adjacent to all the bead vertex and hence odegree of both the vertex is $\mathrm{m}$. At the first stage oidegree is $m-1$ for both the vertices.

Therefore $\operatorname{os}_{2} \boldsymbol{D}_{1}\left(N K_{m}\right)=2(m-1)$.

At the second stage, any one of the vertices is shifted to the dominating set, idegree of the other vertex is 0 and odegree is $m+1$, and oidegree is $m+1$.

Therefore $\operatorname{sos}_{2} D\left(N K_{m}\right)=2(m-1)+(m+1)=3 m-1$.

\section{Energy curves of Necklace}

From the graphs given below it is evident that the flow of oShearEnergies of the with respect to first type is a continuous flow but with second kind of domination it starts with a very high value.
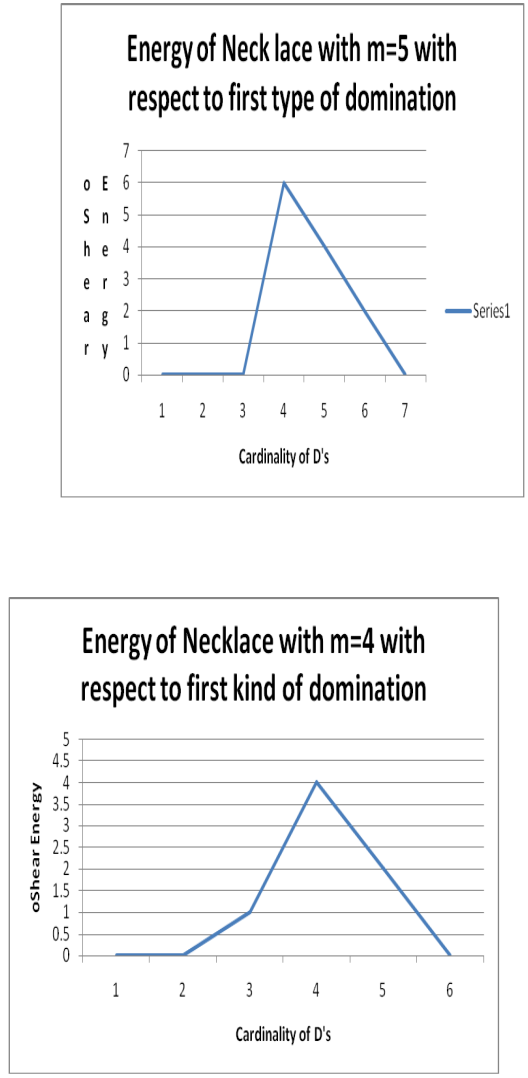

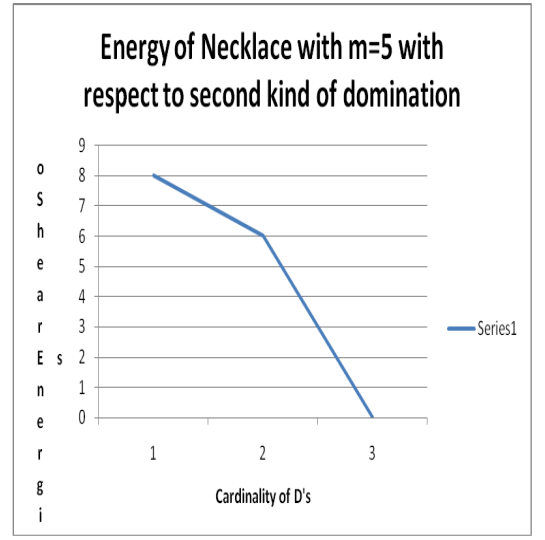

\section{6. soShearEnergy of permutation of a necklace}

Theorem 6.1. Let $\pi$ be a permutation on $p$ symbols $V=$ $\left\{a_{1}, a_{2}, \ldots, a_{p}\right\}$ given by a transposition $\left(a_{1} a_{p}\right)$, where $\mid a_{i+1}-$ $a_{i} \mid=c, c>0$. Then

(i) (a) $\operatorname{sos} \varepsilon_{N K_{m}}\left({ }^{1} D\right)=n^{2}+n+1$ where $n=\frac{m}{2}$ when $m$ is even

(b) $\operatorname{sos} \varepsilon_{N K_{m}}\left({ }^{1} D\right)=(m+1)+\operatorname{sos} \varepsilon_{N K_{m-2}}\left({ }^{1} D\right)$ when $m$ is odd and $\operatorname{sos} \varepsilon_{N K_{1}}\left({ }^{1} D\right)=2$

(ii) $\operatorname{sos} \varepsilon_{N K_{m}}\left({ }^{2} D^{c}\right)=3 m-1$.

Proof. Let $\pi$ be a permutation on p symbols $V=\left\{a_{1}, a_{2}, \ldots, a_{p}\right\}$ given by a transposition $\left(a_{1} a_{p}\right)$, where $\left|a_{i+1}-a_{i}\right|=c, c>0$. Then by the theorem $3.2 \pi$ realizes a necklace $N K_{m}$ with $m=a_{p}-a_{1}-1=p-2$ number of beads and $a_{1} a_{p}$ as rope. For a permutation graph a minimal dominating set can be established using the algorithm given in [2].

The possible minimal dominating sets are $D=\left\{a_{1}\right\}$ or $\left\{a_{p}\right\}$ and $D=\left\{a_{2}, a_{3}, \ldots, a_{p-1}\right\}$.

If $D=\left\{a_{1}\right\}$ or $\left\{a_{p}\right\}$ then $D$ contains the vertices of the rope and hence it is the domination of first kind by Definition 4.1.Therefore by Lemma $4.2 \operatorname{sos}_{N K_{m}}\left({ }^{1} D\right)=n^{2}+n+1$ where $n=\frac{m}{2}$ when $\mathrm{m}$ is even and $\operatorname{sos}_{N K_{m}}\left({ }^{1} D\right)=(m+1)+$ $\operatorname{sos} \varepsilon_{N K_{m-2}}\left({ }^{1} D\right)$ when $\mathrm{m}$ is odd and $\operatorname{sos} \varepsilon_{N K_{1}}\left({ }^{1} D\right)=2$.

If $D=\left\{a_{2}, a_{3}, \ldots, a_{p-1}\right\}$ then $\mathrm{D}$ is the domination of second kind. Hence $\operatorname{sos}_{N K_{m}}\left({ }^{2} D^{c}\right)=3 m-1$.

Hence the proof.

\section{References}

[1] Chartrand Gary, Harary Frank, Planar permutation graphs, Ann. Inst. H. Poincaré Sect. B (N.S.), 3(1967), 433-438.

[2] J. Chithra, S.P. Subbiah, V. Swaminathan Domination in Permutation, International Journal of Computing Algorithm, 3(2014), 549-553.

[3] R. Chitra, S.P. Subbiah, A Study of harmonious Coloring, Labeling and Domination in Graph Structures, 2017, 1219.

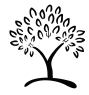


[4] Jeyakokila S.P.,Sumathi P. Energy of set of vertices-A computational method, IJMSEA, 7(III)(2013), 137-148.

[5] Jeyakokila S.P. and Sumathi P., A note on soEnergy of Cocktailparty and crown graphs, International Journal of Applied Science and Mathematics, 3(1)(2008), 23-28.

[6] Jeyakokila, S.P.; Sumathi, P. A note on soEnergy of stars, bistars and double stars graphs, Bull. Int. Math. Virtual Inst., 6(1)(2016), 105-113.

[7] Hedetniemi, S. T, Laskar, R. C, Bibliography on domination in graphs and some basic definitions of domination parameters, Discrete Math., 86(1-3)(1990), 257-277.

[8] Even. S, Pnueli. A, Lempel. A, Permutation graphs and transitive graphs, J. Assoc. Comput. Mach., 19(1972), 400-410.

[9] Uehara, Ryuhei, Valiente, Gabriel, Linear structure of bipartite permutation graphs and the longest path problem, Inform. Process. Lett. 103(2)(2007), 71-77.

[10] M.Kryszkiewiecz, Rules in incomplete information systems, Information Sciences, 113(1999), 271-292.

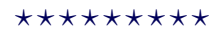

$\operatorname{ISSN}(\mathrm{P}): 2319-3786$

Malaya Journal of Matematik

$\operatorname{ISSN}(\mathrm{O}): 2321-5666$

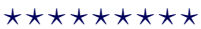

